\title{
Outcomes of Conductive Keratoplasty Combined with Corneal Crosslinking in Advanced Ectatic Corneal Disease
}

This article was published in the following Dove Press journal: Clinical Ophthalmology

\author{
Mazen M Sinjab $\mathbb{D}^{1,2}$ \\ Roy S Rubinfeld ${ }^{3,4}$ \\ Kirsten Wagner ${ }^{5,6}$ \\ Edward C Parsons Jnr $\mathbb{D}^{7}$ \\ Arthur B Cummings $\mathbb{D}^{8}$ \\ Michael W Belin ${ }^{9}$ \\ 'Dr. Mazen Eye Clinic, Medcare Hospitals \\ and Centres, Dubai, United Arab \\ Emirates; ${ }^{2} \mathrm{Al}$ Zahra Medical Group, \\ Damascus, Syria; ${ }^{3}$ Department of \\ Ophthalmology, Georgetown University \\ Medical School/Washington Hospital \\ Center, Washington, DC, USA; ${ }^{4}$ Re: \\ Vision Private Practice, Rockville, MD, \\ USA; ${ }^{5}$ Department of Ophthalmology, \\ Medstar Georgetown University \\ Hospital, Washington, DC, USA; \\ ${ }^{6}$ Department of Ophthalmology, Medstar \\ Washington Hospital Center, \\ Washington, DC, USA; ${ }^{7} \mathrm{CXL}$ \\ Ophthalmics, Encinitas, CA, USA; \\ ${ }^{8}$ Wellington Eye Clinic, Dublin, Ireland; \\ ${ }^{9}$ Department of Ophthalmology, \\ University of Arizona, Tucson, AZ, USA
}

Purpose: To assess the effectiveness of a novel treatment for patients with advanced corneal ectasia and loss of visual acuity (VA). Conductive keratoplasty (CK) is performed to improve VA followed by epithelium-on (epi-on) corneal crosslinking (CXL) to stabilize the cornea after CK.

Methods: Retrospective, exploratory cohort study. Patients with keratoconus or postsurgical ectasia and best spectacle-corrected distance VA $($ CDVA $) \leq 20 / 40$ were included. Conductive keratoplasty was performed (ViewPoint CK System, Refractec, Inc., Bloomington, MN); followed a day later by epi-on CXL (CXLUSA/CXLO, Bethesda, MD/CXLO Encinitas, CA). Measures included uncorrected distance visual acuity (UDVA) and CDVA, as well as refractive and tomographic measures and tomographic indices.

Results: Data from 50 eyes of 45 patients were analyzed. Mean follow-up was $15.1 \pm 12.2$ months (range: 2 to 51). Overall, UDVA and CDVA improved postoperatively. Subjective refraction and tomographic metrics did not show consistent changes, but changes in tomographic indices were associated with treatment follow-up time. At the 1-year visit, mean UDVA significantly improved over baseline $(P=0.009)$ by approximately 3 lines; mean CDVA improved significantly $\left(\mathrm{P}=10^{-5}\right)$ by approximately 2 lines. No eye lost lines of CDVA. Change in the Index of Surface Variance (ISV) was associated with treatment, and the D-Index trended over follow-up time.

Conclusion: Conductive keratoplasty with a proprietary epi-on CXL treatment improved vision in patients with advanced ectasia This CK/epi-on CXL treatment offers the possibility of improved VA for patients with compromised vision due to ectasia.

Keywords: conductive keratoplasty, CK, corneal crosslinking, crosslinking, keratoconus, ectasia, thermokeratoplasty

\section{Introduction}

Keratoconus $(\mathrm{KC})$ and post-surgical ectasia are ectatic corneal diseases characterized by posterior and anterior conical steepening of the cornea with progressive corneal thinning and distortion, and a gradual loss of best spectacle-corrected distance visual acuity (CDVA). Continued progression of the disease can lead to the need for corneal transplantation, which has substantial negative impacts including lifetime economic burden ${ }^{1}$ and risk of rejection/transplant failure. ${ }^{2}$ In addition, corneal instability may still progress, resulting in further loss of CDVA. ${ }^{3}$

Corneal crosslinking (CXL) treatment uses UVA-activated riboflavin to induce protein and matrix crosslinking that strengthens the mechanical properties of the cornea. This
Correspondence: Roy S Rubinfeld

313I Connecticut Ave, NW, \#2809,

Washington, DC, 20008, USA

Email Doctor@revisedeye.com 
stabilizes the cornea and limits the progression of corneal ectasia. ${ }^{4}$ When performed early in the progression of corneal ectasia, spectacles or contact lenses may be sufficient to provide excellent CDVA. However, for patients with significant loss of CDVA due to corneal ectasia, an evolving area of research involves combining CXL with an adjunctive refractive procedure to improve CDVA as well as stabilize the ectatic cornea. ${ }^{5} \mathrm{CXL}$ is generally very effective at stopping progressive ectatic vision loss. Small improvements in mean vision upon treatment have been seen in epi-off $\mathrm{CXL}^{6}$ and in an epion procedure. ${ }^{7}$ For patients with substantial vision impairment due to advanced ectatic disease, we sought to add an adjunctive procedure (CK) to improve their visual function.

Adjunctive refractive procedures have their own liabilities. Customized laser (wavefront and topography guided) vision treatments ${ }^{8-10}$ and transepithelial phototherapeutic and keratectomy (TE-PTK) ${ }^{11-13}$ improve visual acuity (VA), but require further mechanical or laser disruption of, or tissue removal from, already compromised corneas. Intracorneal ring segment (ICRS) implantation, ${ }^{14-17}$ may result in ring segment extrusion, corneal neovascularization, infectious keratitis and ring segment migration. ${ }^{18}$ Conductive keratoplasty is less disruptive of the cornea than other refractive procedures, however regression of the effect has been reported from 3-6 months post-procedure. ${ }^{19}$ Regression of effect has been known to be a limiting factor in thermal refractive procedures for decades. ${ }^{20,21}$

Two recent case reports have described a combined CK and transepithelial (epi-on) CXL procedure that produced a stable improvement in CDVA for at least 1 year. ${ }^{22,23} \mathrm{We}$ have worked to evolve this CK procedure using customized tomographic and topographic guided CK in conjunction with an optimized apical spot protocol. Subjects consisted of a small cohort of study patients from a clinical trial of a novel epi-on CXL system (CXLUSA, Bethesda, MD/ CXLO, Encinitas, CA epi-on protocol). These subjects also underwent $\mathrm{CK}$ to treat their moderate to severe loss of CDVA due to keratoconus or post-LASIK ectasia. This combination of CK with epi-on CXL is minimally invasive and avoids removal (excimer laser) or disruption (ICRS, epi-off CXL) of tissue from an already weakened cornea; as well as complications ${ }^{24}$ associated with intra-corneal implantation of foreign bodies (ICRS). The combination of CK and epion CXL utilizes an off-label application of the CK System (ViewPoint ${ }^{\mathrm{TM}}$ CK System, Refractec, Inc, Bloomington, $\mathrm{MN}$ ). Epi-on CXL was performed as part of Institutional Review Board (IRB)-approved studies.
This is a retrospective, exploratory analysis to assess whether this $\mathrm{CK}$ technique, used with a proprietary epi-on CXL procedure, results in improved VA beyond the 3-6 month period identified in previous studies for regression of CK. ${ }^{25}$ Tomographic parameters were also assessed throughout follow-up to identify other potential correlates to clinical efficacy.

\section{Methods}

\section{Study}

The data for this exploratory analysis come from "A study of collagen crosslinking with ultraviolet-A in asymmetric corneas" (Clinical Trials.gov identifier NCT01097447 and NCT01956474), in which a subset of patients, with more advanced disease, received CK treatment in addition to CXL. The study was initiated in 2012 under Institutional Review Board-approved protocols at Washington Eye Physician and Surgeons, Chevy Chase, MD (IRBCo, Buena Park, CA) and then continued at Re:Vision-Roy Rubinfeld, MD, Rockville, MD and Fairfax, Virginia (Quorum IRB, Seattle, Washington). Data collection was over a 5-year period from July, 2013 to July, 2018. All procedures adhered to the tenets of the Declaration of Helsinki. Informed consent was obtained from all participants before any study procedures were performed.

\section{Patients}

Patients with preoperative CDVA $\leq 20 / 40$ were included in the cohort that received CK in addition to CXL. Data were included in this analysis if the patient had a diagnosis of keratoconus or post-surgical ectasia, had received the combined CK-CXL procedure and had completed the 2-month, or later, postoperative exam. Patients were excluded from the original study if they were found to have a corneal thickness of $<325 \mu \mathrm{m}$, severe corneal opacity, a history of corneal hydrops, herpetic keratitis or other comorbidity. Patients who used rigid or hybrid contact lenses within 4 weeks of the procedure or had previously received penetrating keratoplasty, radial keratotomy or CXL procedures were also excluded. Patients presenting with early-stage ectasia and good visual function did not require CK and received epi-on CXL as a stand-alone procedure and were not included in this report.

\section{Preoperative Baseline Evaluation}

Preoperative evaluation included measurement of uncorrected distance VA (UDVA) and CDVA (defined as best spectacle- 
corrected visual acuity) by high contrast Snellen charts, subjective astigmatism, spherical equivalent (SEQ), Equivalent K-readings 65\% (EKR65 1 and 2) and EKR global range from Holladay report, mean $\left(\mathrm{K}_{\mathrm{m}}\right)$ and maximum keratometry $\left(\mathrm{K}_{\mathrm{Max}}\right)$ of the anterior corneal surface, Belin/Ambrosio enhanced ectasia total deviation value (D index) and index of surface variance (ISV) (Pentacam HR OCULUS, Optikgeräte GmbH Wetzlar, Germany), slit lamp biomicroscopy, intraocular pressure (IOP) by Goldmann tonometry and dilated fundus exam. The surgical techniques, objectives, side effects and possible risks were discussed with the patients and appropriate consent forms were signed by the patients or assent forms were signed for minors by their parents or legal guardians.

\section{Surgery}

All surgeries were performed under topical anesthesia by one surgeon (RSR). Conductive keratoplasty was performed by applying controlled radiofrequency energy with the ViewPoint ${ }^{\mathrm{TM}}$ CK System using an optimized apical spot placement method. An inked optical zone corneal marker of $\sim 3.5 \mathrm{~mm}$ diameter, centered on the cornea relative to the limbus, provided reference points. The patient's preoperative 4-map Refractive Pentacam printout was displayed appropriately in the surgical view to identify the placement of the initial CK spots (both posterior and anterior corneal surface map data were visualized). A preliminary plan was devised before each surgery, based on numerical keratometric values on sagittal curvature as well as posterior elevation maps. Ideally the apical region on the sagittal map matched the maximum posterior elevation. When this was not the case, a small adjustment of 1-2 mm toward the area of maximum posterior elevation was considered. The surgeon placed spots at an optical zone of roughly 3-4 mm (ie $1.5-2 \mathrm{~mm}$ from the corneal center) in the meridian of the steepest part of the cone (Figure 1).

During the $\mathrm{CK}$ procedure, the Mastel operating keratoscope (Mastel Precision Surgical Instruments, Inc., Rapid City, SD) or an operating microscope ring light (Visx/ Johnson and Johnson Vision, Jacksonville, FL) was projected onto the cornea to monitor the anterior curvature of the cornea. Gentle pressure was applied to the cornea with a blunt instrument or angled part of the sterile $\mathrm{CK}$ probe in order to find the optimal spot position that could be observed to "round up" an oval or irregular corneal shape, providing feedback via surgeon-observed changes induced in the ring light reflection by roughly previewing the effect of a $\mathrm{CK}$ spot (Figure 2).

These techniques allowed real-time intraoperative monitoring of the $\mathrm{CK}$ effects on the anterior corneal curvature. Changes in the corneal curvature were easily visible and evaluated immediately post-CK by serial Pentacam imaging (Figure 3). Typical immediate postoperative slit lamp appearance after peri-apical CK spots are placed is shown in Figure 4. Serial, sagittal, Pentacam scans and difference maps were viewed to assess whether more spots might be needed (Figure 5).

The goal for the initial CK spot placement was significant overcorrection. This was seen in approximately two-thirds of our cases after placement of the initial set of spots. This can be
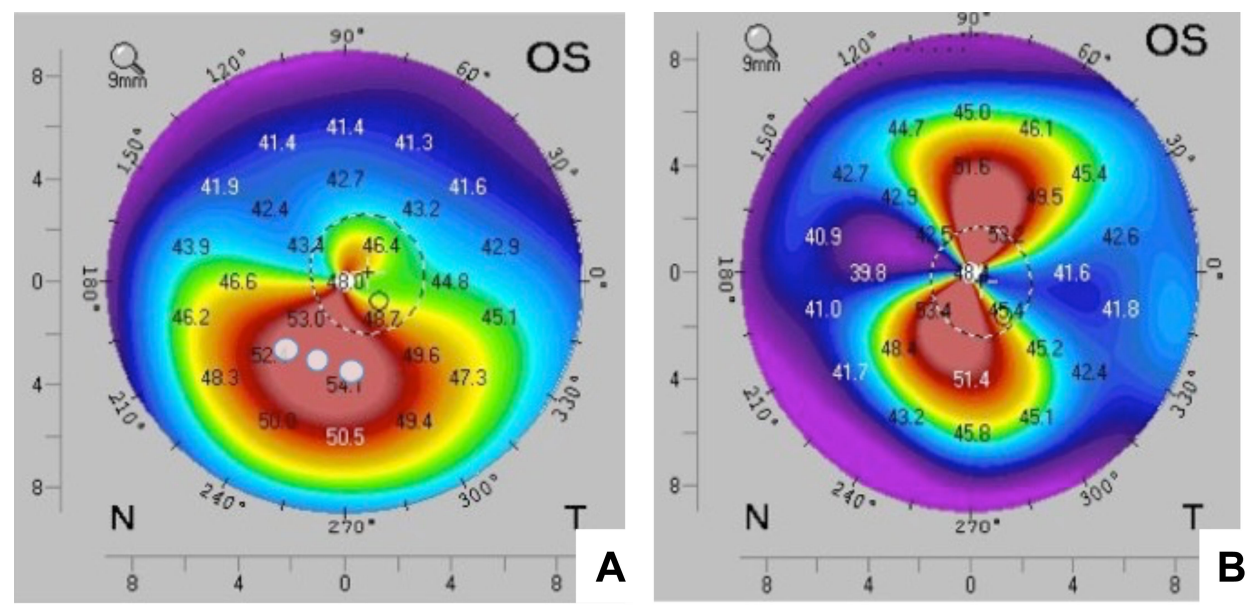

Figure I Example of a Pentacam (front sagittal curvature) scan of a case with an infero-central cone apex (A) directly before and (B) I day after conductive keratoplasty (CK). (A) The 3 white circles indicate the typical placement of the initial CK spots. The yellow circle displays the $\sim 3.5 \mathrm{~mm}$ optical zone. Once the spots are placed, the ring light or keratometer reflections are assessed to determine if the spots had a substantial effect in "rounding up" the irregular reflection. Repeat sagittal Pentacam scans and difference maps are then reviewed to assess if, and where, additional CK spots should be placed to achieve the desired initial overcorrection. (B) Postoperative image displaying the achievement of regular astigmatism (symmetrical bowtie) of the cornea after CK. K-values are noted on the images. 


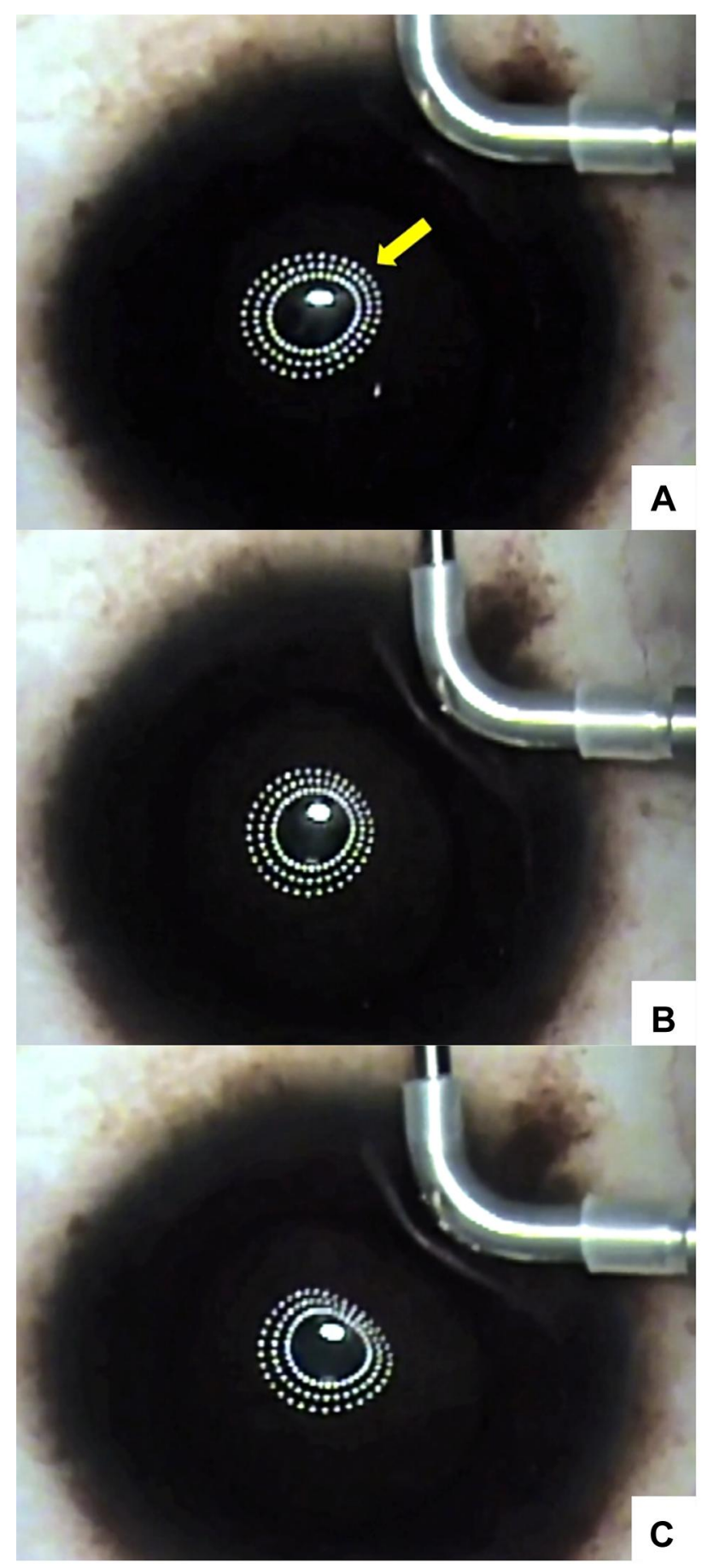

Figure 2 (A) Preoperative surgeon view (upside down) through operating microscope before application of pressure on the cornea with blunt instrument (the back end of the conductive keratoplasty (CK) probe). Note reflected light images form a teardrop shape with the more acute curve pointing toward the 2 o'clock position (yellow arrow). Pressing on the cornea peripherally at this axis can help determine where the first few CK spots should be applied. (B) Pressure at this 2 o'clock position with the back end of the CK probe "rounds up" the reflected light images, demonstrating a more spherical cornea with less irregular astigmatism. This helps confirm where the first spots should be placed in this eye. (C) Additional pressure with the back of the CK probe shows even more effect on the reflected ring lights and corneal shape. Because early regression is expected after CK, this image shows roughly the degree of over-correction initially desired immediately after CK.

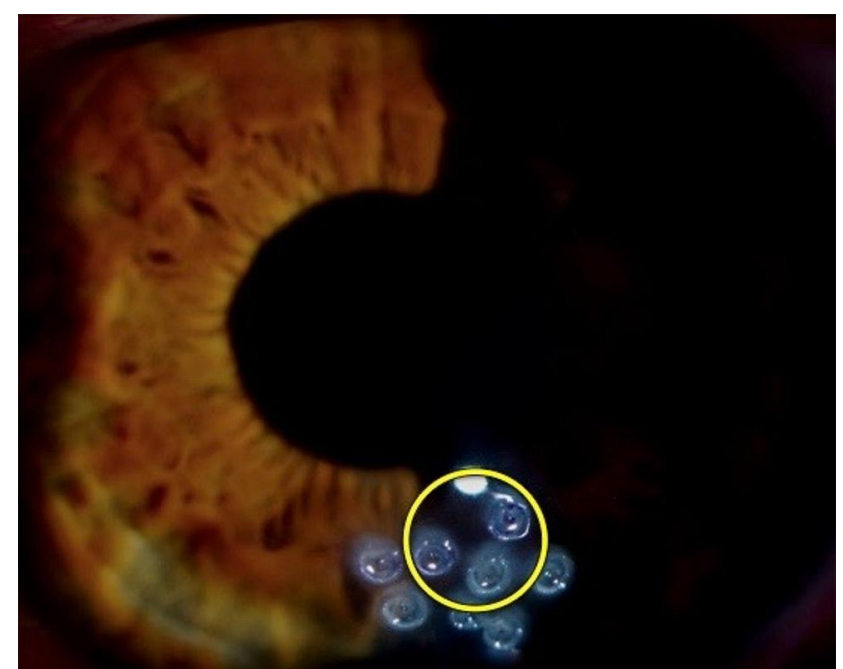

Figure 3 Slit lamp image of initial conductive keratoplasty (CK) spot placement at 3.5 optical zone (enclosed within yellow circle). These three spots were placed based on the Pentacam scan and ring reflections as described in the text. After a repeat Pentacam scan, the anterior postoperative image showed more astigmatic regularity but not the desired overcorrection. Additional spots (seen outside the yellow circle) were placed near the initial CK spots to achieve the desired overcorrection.

observed in real time through the operating microscope ${ }^{\mathrm{A}}$ (see Supplemental Digital Content) in which a single CK spot can be seen to markedly flatten the steep "teardrop" end of the reflected ring light. This is also visible in the bottom image in Figure 2. In the remaining one-third of treated eyes, an additional 1-3 CK spots were placed, based on the Pentacam scans obtained immediately after the first set of spots, to obtain the desired initial overcorrection (Figures 3 and 4).

In cases where the steep area of the cornea was relatively thin $(<375-400 \mu \mathrm{m})$, we applied the CK probe tip at an oblique angle to avoid perforating the full thickness of the cornea. Attempts were made to avoid overlapping or confluent CK spots.

Because immediate thermal keratoplasty effects quickly regress (Figure 5), we aimed for substantial overcorrection, especially in advanced, steep cones. Epi-on CXL was scheduled for the next day using the CXLUSA epi-on protocol. ${ }^{7,23,26,27}$

All patients used generic polymyxin $b$ sulfate and trimethoprim ophthalmic solution qid and generic prednisolone acetate $1 \%$ suspension qid for 3 days postoperatively as well as artificial tears as needed.

\section{Postoperative Examinations}

Postoperative examinations 1 and 2 were performed at 1 day and 1 week to address procedure safety. Study endpoints were assessed at up to 6 additional follow-up examinations to assess 

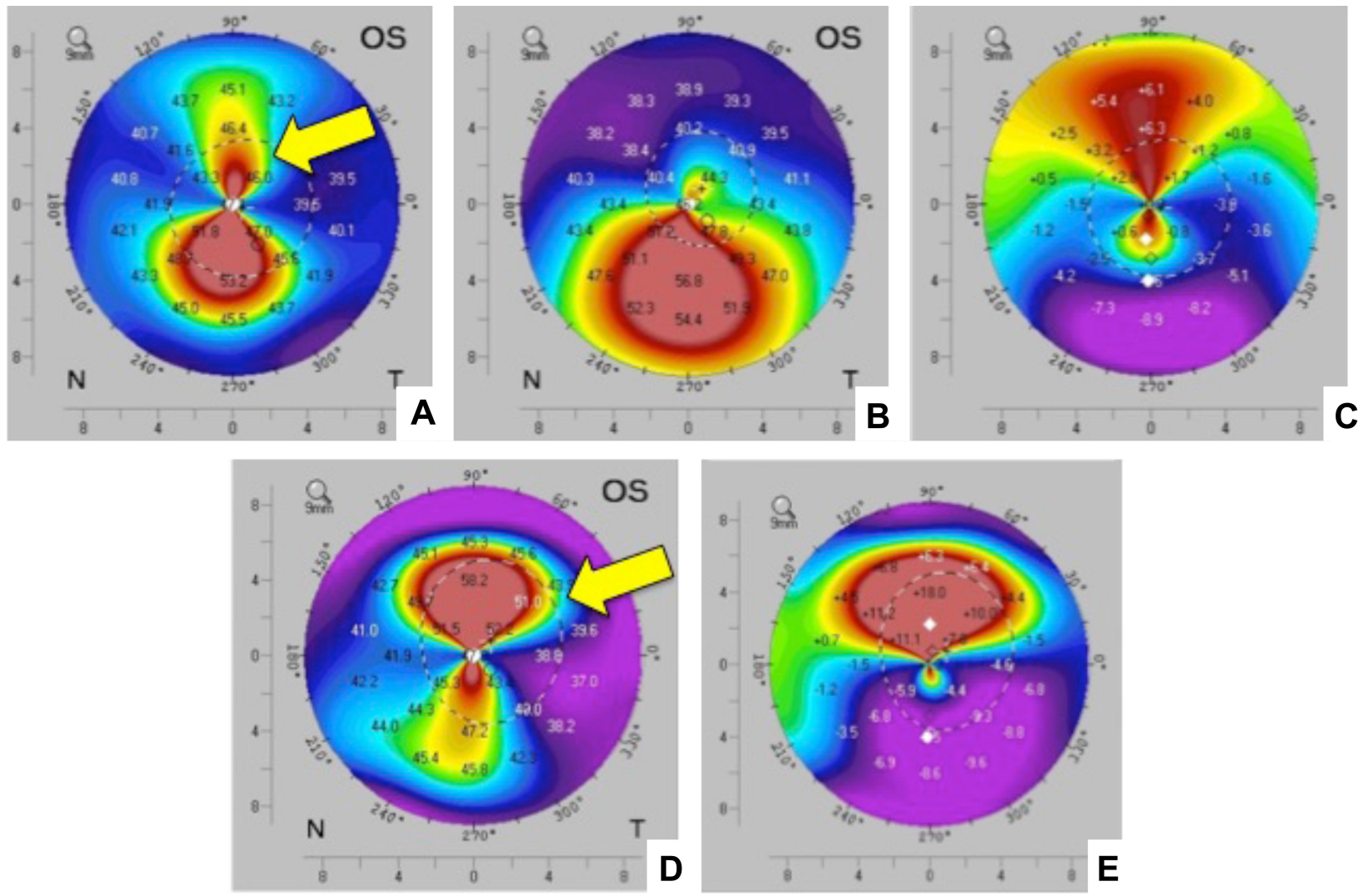

Figure 4 Upper row: Anterior sagittal curvature difference map. (A) Postoperative map showing substantial reduction in corneal irregularity (yellow arrow) compared to (B) preoperative infero-central cone at $\sim 6$ o'clock and (C) difference map. Lower row: Serial anterior sagittal curvature Pentacam scans in the same eye in which the initial conductive keratoplasty (CK) effect was substantial but additional CK spots were needed. (D) Map shows an additional effect with some visible overcorrection (yellow arrow). (E) The digital subtraction (difference map) showing the difference between the preoperative scan and results of the second set of CK spots. The serial Pentacam images allow us to assess the adequacy of the effect and determine whether further CK spots are warranted.
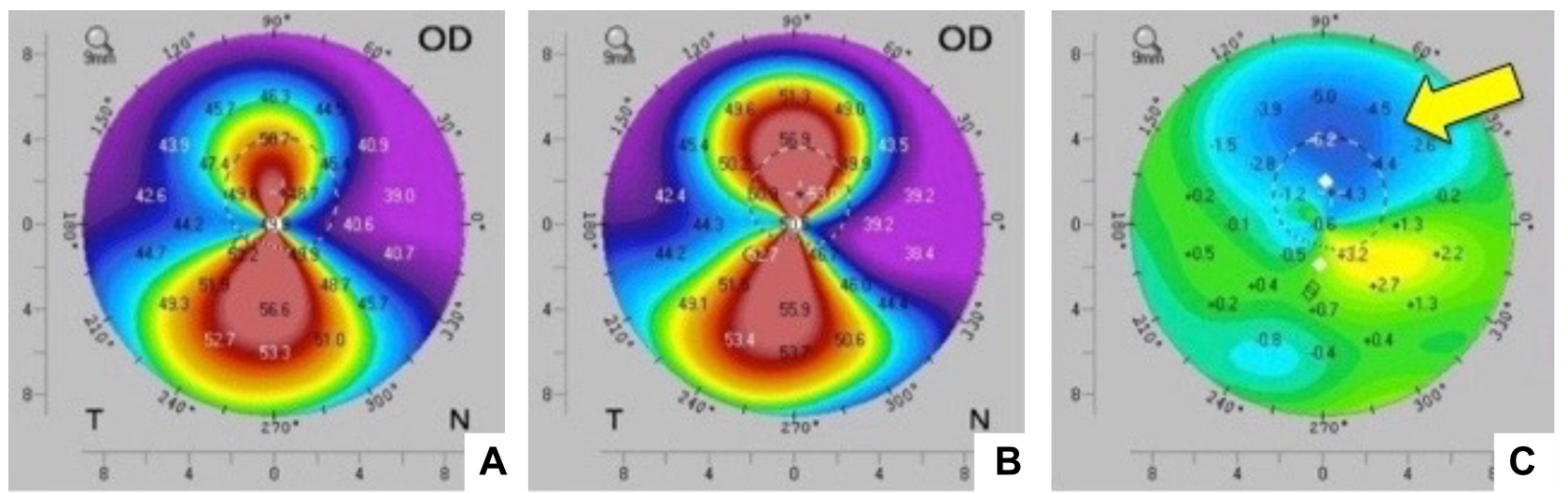

Figure 5 Pentacam scans (front or sagittal curvature) of early regression of conductive keratoplasty (CK) effect over $\sim 26$ hours. (A) The map shows a case $\sim 26$ hrs after CK treatment compared to (B), a scan taken immediately after CK treatment, but before any crosslinking treatment. (C) Demonstrates the difference between $A$ and $B$ (digital subtraction map) showing $\sim 6$ diopters of early regression since the CK treatment (yellow arrow).

procedure safety and efficacy: Visit 3 at $1 \mathrm{~m}$ postoperative, Visit 4 at $2 \mathrm{~m}-4.5 \mathrm{~m}$, Visit 5 at $4.5 \mathrm{~m}$ to $10 \mathrm{~m}$, Visit 6 at $10 \mathrm{~m}$ to $16 \mathrm{~m}$, Visit 7 at $16 \mathrm{~m}$ to $32 \mathrm{~m}$ and Visit 8 at $33 \mathrm{~m}$ to $53 \mathrm{~m}$. Visits 3-8 included routine ophthalmologic and fundoscopic examinations, IOP and the study endpoints: UDVA, CDVA, subjective astigmatism, spherical equivalent (SEQ), Equivalent K-readings 65\% (EKR65 1 and 2) and EKR global range from Holladay report, mean $\left(\mathrm{K}_{\mathrm{m}}\right)$ and maximum 
keratometry $\left(\mathrm{K}_{\mathrm{Max}}\right)$ of the anterior corneal surface, Belin/ Ambrosio enhanced ectasia total deviation value (D index), and index of surface variance (ISV). Visits 6-8 were optional for the subjects.

\section{Statistical Analysis}

Measurements were tabulated by visit and descriptive statistics were summarized (mean and standard deviation, mean and 95\% confidence interval (CI) of change from baseline). The primary analysis was of change from baseline in VA (CDVA and UDVA) at 1-year postoperative (Visit 6). Snellen values were converted to $\operatorname{logMAR}$ units and a paired $t$-test was performed, comparing the baseline and Visit 6 measurements (Excel version 16.22, Microsoft, Redmond, WA). Approximate Snellen values are included in parentheses after $\log$ MAR values.

We assessed trends in the 11 visual acuity and tomographic parameters measured over time, and the effect of treatment on each. Given the variations in the number and timing of follow-up visits, we performed an exploratory multiple regression analysis to examine the effects of time and treatment on each parameter. A linear model was applied to the data (Using R v3.6.2, R Core Team) for each continuous parameter. For example, for CDVA:

$$
\operatorname{CDVA}-\operatorname{CDVA}(0)=\mathrm{a} * \text { treatment }+\mathrm{b} * \text { time }
$$

The change from baseline CDVA, expressed in terms of:

Treatment $=$ a dummy variable equal to 0 for baseline measurements, and 1 for postoperative measurements,

Time $=$ a continuous metric of duration between procedure and examination.

The regression analysis computed least-squared error estimates of coefficients $a$ and $b$, the predicted effect of treatment and of time, respectively. The analysis also returns a $P$-value for each coefficient, indicating the likelihood that the effect size is greater than zero. The regression analysis was repeated for the subset of patients with a diagnosis of keratoconus, and where only one eye (with worse baseline CDVA) was used per patient, to assess just the keratoconus population with equal weighting.

\section{Results}

A total of 50 eyes from 45 patients met the study criteria. Of these patients, $22.2 \%$ were female, $77.8 \%$ male; the mean age was $33.2 \pm 12.0$ years (range 18-68 years). There were 42 eyes (84\%) with $\mathrm{KC}$ and 8 eyes $(16 \%)$ with postsurgical ectasia. Twelve of the 50 eyes were from patients over the age of 40 years and presbyopic.
All patients had a preoperative baseline examination. The distribution of the number of follow-up efficacy evaluations per-patient contributing to the analysis is shown in Table 1. Visit Numbers 4, 5, 6, 7, and 8 were attended by $36,31,28,9$, and 7 patients, respectively. The average timing of the final follow-up visit was $15.1 \pm 12.2$ (mean \pm SD) months post-surgery (range 2-51 months).

At baseline $(n=50)$, the mean central corneal thickness (CCT) was $473 \pm 46 \mu \mathrm{m}$ (range: $374-574 \mu \mathrm{m}$ ) and the mean thickness of subject corneas at their thinnest point (TCT) was $418.8 \pm 55.5 \mu \mathrm{m}$ (range: $292-528 \mu \mathrm{m}$ ). Mean preoperative and postoperative values for UDVA, CDVA, subjective astigmatism, SEQ, K values, D-index and ISV are expressed as mean $\pm \mathrm{SD}$ in Table 2. Mean changes from baseline for postoperative measures are detailed in Table 3, along with 95\% confidence intervals.

The exploratory regression analyses fit measurements from 185 patient-visits for each of the 11 parameters. In each case, F-tests returned $P$-values uniformly $<2 \times 10^{-16}$, indicating the overall significance of the model. The treatment and time coefficients of best fit and their associated $P$ values are listed in Table 4 for the four parameters that demonstrated significant change from baseline (UDVA, CDVA, D index and ISV).

The exploratory regression analysis was repeated on the subset of 37 patients with keratoconus. For the 5 patients contributing 2 eyes to the full analysis, only the eye with worse preoperative CDVA was used. The results were similar to the full analysis set, with significant effects only in the same four parameters. Those results are summarized in Table 5.

\section{Visual Acuity}

By-visit mean VAs are detailed in Table 2; changes from baseline in mean VAs in Table 3 and Figure 6. At baseline, mean UDVA was $\log$ MAR $1.2 \pm 0.6$ (20/

Table I Distribution of Number of Follow-Up Efficacy Evaluations Analyzed

\begin{tabular}{|c|c|}
\hline Number of Visits & Number of Patients \\
\hline 1 & 4 \\
2 & 21 \\
3 & 9 \\
4 & 6 \\
5 & 3 \\
6 & 2 \\
\hline Total & 45 \\
\hline
\end{tabular}


Table 2 Summary of Baseline and Postoperative Vision and Tomographic Parameters Measured (Mean \pm SD)

\begin{tabular}{|c|c|c|c|c|c|c|c|}
\hline $\begin{array}{l}\text { Visit(Months) } \\
\text { Mean } \pm \text { SD (n) }\end{array}$ & $\begin{array}{l}\text { Baseline } \\
0 \pm 0(50)\end{array}$ & $\begin{array}{c}\text { Visit } 3 \\
\mathrm{I} .3 \pm 0.5(24)\end{array}$ & $\begin{array}{c}\text { Visit } 4 \\
5.3 \pm 0.2(36)\end{array}$ & $\begin{array}{c}\text { Visit } 5 \\
8.6 \pm 0.3(31)\end{array}$ & $\begin{array}{c}\text { Visit } 6 \\
\text { | } 3.8 \pm 0.3(28)\end{array}$ & $\begin{array}{c}\text { Visit } 7 \\
27.5 \pm 1.2(10)\end{array}$ & $\begin{array}{c}\text { Visit } 8 \\
39.5 \pm 2.4(7)\end{array}$ \\
\hline UDVA (logMAR) & $1.2 \pm 0.6(47)$ & $0.6 \pm 0.3(24)$ & $0.9 \pm 0.5(37)$ & $0.9 \pm 0.5(3 \mathrm{I})$ & $0.9 \pm 0.4(27)$ & $0.9 \pm 0.3(9)$ & $I \pm 0.4(7)$ \\
\hline CDVA (logMAR) & $0.5 \pm 0.2(50)$ & $0.3 \pm 0.2(23)$ & $0.3 \pm 0.2(37)$ & $0.3 \pm 0.1(3 I)$ & $0.3 \pm 0.1(28)$ & $0.3 \pm 0.1(10)$ & $0.3 \pm 0.2(7)$ \\
\hline Subjective Astigmatism (D) & $-1.7 \pm 4.1(48)$ & $-0.4 \pm 3.6(23)$ & $-1.6 \pm 4.1(37)$ & $-1.9 \pm 4.1(31)$ & $-2.3 \pm 3.9(28)$ & $-4.4 \pm 1.1(10)$ & $-3.4 \pm 1.8(7)$ \\
\hline SEQ (D) & $-4.2 \pm 4.6(48)$ & $-3.5 \pm 4(23)$ & $-3.6 \pm 4.6(37)$ & $-3 \pm 5.3(3 I)$ & $-3.9 \pm 4.6(28)$ & $-3.3 \pm 2.5(10)$ & $-4.7 \pm 3.8(7)$ \\
\hline EKR65 KI (4.5mm) & $47 \pm 8(44)$ & $46 \pm 6(16)$ & $47 \pm 8(35)$ & $48 \pm 8(29)$ & $47 \pm 8(25)$ & $49 \pm 8(10)$ & $50 \pm 9(7)$ \\
\hline EKR65 K2 (4.5mm) & $52 \pm 8(44)$ & $52 \pm 6(16)$ & $51 \pm 7(35)$ & $52 \pm 7$ (29) & $50 \pm 7(25)$ & $53 \pm 8(10)$ & $52 \pm 9(7)$ \\
\hline EKR Global Range & $24 \pm 10(44)$ & $24 \pm 15(16)$ & $23 \pm 11(35)$ & $24 \pm$ II (29) & $22 \pm 8(25)$ & $24 \pm 8(10)$ & $22 \pm 10(7)$ \\
\hline $\mathrm{K}_{\mathrm{m}}(\mathrm{D})$ & $52 \pm 11(44)$ & $52 \pm 6(15)$ & $52 \pm 8(35)$ & $53 \pm 7(28)$ & $52 \pm 7(25)$ & $55 \pm 8(10)$ & $55 \pm 10(7)$ \\
\hline $\mathrm{K}_{\operatorname{Max}}(\mathrm{D})$ & $65 \pm 11(44)$ & $66 \pm 14(22)$ & $64 \pm 10(37)$ & $64 \pm 10(31)$ & $64 \pm 10(28)$ & $67 \pm 11(10)$ & $66 \pm 11(7)$ \\
\hline$D$ index & $16 \pm 9(44)$ & $12 \pm 5(16)$ & $16 \pm 9(34)$ & $16 \pm 6(29)$ & $17 \pm 7(25)$ & $20 \pm 10(9)$ & $22 \pm 13(7)$ \\
\hline ISV & $149 \pm 49(44)$ & $104 \pm 38(16)$ & $123 \pm 40$ & $129 \pm 35(29)$ & $134 \pm 49(25)$ & $138 \pm 38(10)$ & $138 \pm 52(7)$ \\
\hline
\end{tabular}

Note: Endpoint parameters expressed as Mean \pm Standard Deviation (n).

Abbreviations: SD, standard deviation; $n$, number of eyes; UDVA, uncorrected distance visual acuity; CDVA, corrected distance visual acuity; D, diopters; SEQ, spherical equivalent; EKR, equivalent keratometric reading; EKR KI, minimum equivalent keratometric reading; EKR K2, maximum equivalent keratometric reading; $K_{m}$, mean keratometric reading on the anterior corneal surface; $\mathrm{K}_{\operatorname{Max}}$, maximum keratometric reading on the anterior corneal surface; ISV, index of surface variance.

Table 3 Mean Parameter Changes from Baseline by Visit (Lower 95\% Cl, Upper 95\% Cl)

\begin{tabular}{|c|c|c|c|c|c|c|}
\hline $\begin{array}{l}\text { Visit (Months) } \\
\text { Mean } \pm \text { SD (n) }\end{array}$ & $\begin{array}{c}\text { Visit } 3 \\
1.3 \pm 0.5(24)\end{array}$ & $\begin{array}{c}\text { Visit } 4 \\
3.3 \pm 0.4(36)\end{array}$ & $\begin{array}{c}\text { Visit } 5 \\
6.8 \pm 0.9(31)\end{array}$ & $\begin{array}{c}\text { Visit } 6 \\
12.1 \pm 1.8(28)\end{array}$ & $\begin{array}{c}\text { Visit } 7 \\
26.3 \pm 3.2(9)\end{array}$ & $\begin{array}{c}\text { Visit } 8 \\
38.3 \pm 6(7)\end{array}$ \\
\hline UDVA (LogMAR) & $-0.42(-0.62,-0.22)$ & $-0.29(-0.46,-0.13)$ & $-0.40(-0.6,-0.19)$ & $-0.31(-0.51,-0.10)$ & $0.02(-0.38,0.43)$ & $-0.50(-1.03,0.03)$ \\
\hline CDVA (LogMAR) & $-0.13(-0.20,-0.05)$ & $-0.14(-0.21,-0.07)$ & $-0.16(-0.21,-0.11)$ & $-0.22(-0.30,-0.14)$ & $-0.21(-0.28,-0.13)$ & $-0.27(-0.46,-0.08)$ \\
\hline $\begin{array}{l}\text { Subjective } \\
\text { Astigmatism (D) }\end{array}$ & $-0.95(-1.66,-0.24)$ & $0.08(-0.46,0.63)$ & $-0.06(-0.75,0.62)$ & $0.05(-0.68,0.79)$ & $1.08(-0.05,2.21)$ & $-0.18(-1.16,0.81)$ \\
\hline SEQ (D) & $0.50(-1.24,2.24)$ & $0.20(-0.96,1.36)$ & $1.28(-0.47,3.02)$ & $0.69(-0.26,1.63)$ & $0.46(-1.43,2.35)$ & $1.80(-1.20,4.81)$ \\
\hline EKR65 KI $(4.5 \mathrm{~mm})$ & $-1.21(-3.76,1.33)$ & $0.44(-0.92,1.79)$ & $0.39(-1.28,2.06)$ & $0.95(-1.02,2.93)$ & $0.70(-4.3 \mathrm{I}, 5.7 \mathrm{I})$ & $-0.59(-3.94,2.75)$ \\
\hline EKR65 K2 $(4.5 \mathrm{~mm})$ & $-0.84(-2.67,0.99)$ & $-0.70(-1.67,0.26)$ & $-0.63(-1.79,0.53)$ & $-1.27(-2.90,0.37)$ & $-0.83(-4.16,2.49)$ & $-2.51(-5.23,0.21)$ \\
\hline EKR Global Range & $2.31(-4.18,8.79)$ & $-2.06(-4.69,0.58)$ & $-1.96(-4.93,1.00)$ & $-3.84(-6.94,-0.74)$ & $-3.59(-8.17,1.00)$ & $-3.40(-9.67,2.87)$ \\
\hline $\mathrm{K}_{\mathrm{m}}(\mathrm{D})$ & $0.08(-3.53,3.69)$ & $-0.59(-3.97,2.78)$ & $-0.20(-2.14,1.74)$ & $2.02(-2.61,6.64)$ & $-1.42(-3.32,0.47)$ & $-1.29(-3.93,1.36)$ \\
\hline $\mathrm{K}_{\max }(\mathrm{D})$ & $2.8(-1.0,6.6)$ & $-2.9(-4.9,-0.8)$ & $-2.1(-4.2,0.1)$ & $-1.8(-4.0,0.4)$ & $-2.6(-6.9,1.7)$ & $-3.8(-9.2,1.5)$ \\
\hline$D$ index & $-0.8(-2.6,1.1)$ & $-1.4(-3.2,0.4)$ & $1.5(0.3,2.6)$ & $1.3(-1.3,3.8)$ & $4.2(0.6,7.7)$ & $5.6(0.0,11.1)$ \\
\hline ISV & $-32.6(-55.2,-10.0)$ & $-24.2(-32.5,-15.8)$ & $-24.1(-36.4,-11.9)$ & $-19.3(-35.3,-3.3)$ & $-10.4(-30.1,9.2)$ & $-4.9(-30.5,20.8)$ \\
\hline
\end{tabular}

Note: Endpoint parameters expressed as the mean (lower $95 \% \mathrm{Cl}$, upper $95 \% \mathrm{Cl}$ ) assuming a Gaussian distribution.

Abbreviations: $\mathrm{Cl}$, confidence interval; SD, standard deviation; $\mathrm{n}$, number of eyes; UDVA, uncorrected distance visual acuity; CDVA, best spectacle-corrected distance visual acuity; LogMAR, logarithm of the minimum angle of resolution; D, diopters; SEQ, spherical equivalent; EKR, equivalent keratometric reading; EKR KI, minimum equivalent keratometric reading; EKR K2, maximum equivalent keratometric reading; $\mathrm{K}_{\mathrm{m}}$, mean keratometric reading on the anterior corneal surface; $\mathrm{K}_{\text {Max }}$, maximum keratometric reading on the anterior corneal surface; ISV, index of surface variance.

340 ), with a range of $0.4-2.6$, The mean CDVA was $\log$ MAR $0.5 \pm 0.2(20 / 60)$ with a range of $0.3-1.3$. At 1 year, mean UDVA improved significantly by approximately 3 lines $(P=0.009)$ and mean CDVA improved significantly by approximately 2 lines $\left(P=10^{-5}\right)$. 
Table 4 Results of Regression Analyses for Time and Treatment Effect

\begin{tabular}{|l|l|c|l|c|}
\hline Parameter & $\begin{array}{l}\text { Treatment } \\
\text { Effect }\end{array}$ & $\begin{array}{l}\text { P-value for Overall } \\
\text { Treatment Effect }\end{array}$ & $\begin{array}{c}\text { Time Effect } \\
\text { (per Year) }\end{array}$ & $\begin{array}{c}\text { P-value for Time } \\
\text { Effect }\end{array}$ \\
\hline UDVA (LogMar) & $-0.38 \pm 0.07$ & $6.3 \times 10^{-7}$ & $0.05 \pm 0.05$ & 0.327 \\
\hline CDVA (LogMar) & $-0.15 \pm 0.02$ & $9.2 \times 10^{-9}$ & $-0.03 \pm 0.01$ & 0.067 \\
\hline Subjective Astigmatism (D) & $-0.22 \pm 0.26$ & 0.404 & $0.17 \pm 0.15$ & 0.259 \\
\hline SEQ (D) & $0.49 \pm 0.6$ & 0.413 & $0.18 \pm 0.33$ & 0.593 \\
\hline EKR65 KI (4.5mm) & $-0.02 \pm 0.79$ & 0.984 & $0.36 \pm 0.43$ & 0.406 \\
\hline EKR65 K2 (4.5mm) & $-0.64 \pm 0.52$ & 0.224 & $-0.2 \pm 0.28$ & 0.482 \\
\hline EKR Global Range & $-0.88 \pm 1.42$ & 0.536 & $-0.95 \pm 0.77$ & $0.41 \pm 0.62$ \\
\hline K (D) & $0.02 \pm 1.14$ & 0.987 & $-0.68 \pm 0.6$ & 0.513 \\
\hline K Max (D) & $-0.71 \pm 1.06$ & 0.504 & $2.2 \pm 0.5$ & 0.255 \\
\hline D index & $-1.4 \pm 0.8$ & 0.099 & $7.1 \pm 2.7$ & $3.2 \times 10^{-6}$ \\
\hline ISV & $-28.5 \pm 5.0$ & $6.7 \times 10^{-8}$ & 0.010 \\
\hline
\end{tabular}

Note: $\mathrm{p}$-value is $\operatorname{Pr}(>|\mathrm{t}|)$ for the treatment and time parameters from the regression model: endpoint change $=\mathrm{a}^{*}$ treatment $+\mathrm{b} *$ time.

Table 5 Results of Regression Analyses for Time and Treatment Effect for the Four Parameters Showing Statistically Significant Changes from Baseline. Only Keratoconus Patients Were Included, and Only the Eye with Worse Baseline CDVA if Both Were Treated. $\mathrm{N}=37$ Eyes of 37 Patients

\begin{tabular}{|l|l|l|l|c|}
\hline $\begin{array}{l}\text { Effects in } \\
\text { (Months } \pm \text { SE) }\end{array}$ & $\begin{array}{l}\text { Treatment } \\
\text { Effect }\end{array}$ & $\begin{array}{l}\text { P value for Overall } \\
\text { Treatment Effect }\end{array}$ & $\begin{array}{l}\text { Time Effect } \\
\text { (per Year) }\end{array}$ & $\begin{array}{c}\boldsymbol{P} \text { value for } \\
\text { Time Effect }\end{array}$ \\
\hline UDVA (LogMar) & $-0.35 \pm 0.09$ & $1.13 \times 10^{-4}$ & $0.00 \pm 0.05$ & 0.951 \\
\hline CDVA (LogMar) & $-0.12 \pm 0.03$ & $9.59 \times 10^{-6}$ & $-0.04 \pm 0.01$ & 0.014 \\
\hline D index & $-1.91 \pm 0.96$ & 0.049 & $2.46 \pm 0.51$ & $4.16 \times 10^{-6}$ \\
\hline ISV & $-22.23 \pm 4.98$ & $1.79 \times 10^{-5}$ & $4.35 \pm 2.65$ & 0.103 \\
\hline
\end{tabular}

Note: $P$ value is $\operatorname{Pr}(>|t|)$ for the treatment and time parameters from the regression model: endpoint change $=a^{*}$ treatment $+b^{*}$ time.

Abbreviations: UDVA, uncorrected distance visual acuity; CDVA, best spectacle-corrected distance visual acuity; LogMAR, logarithm of the minimum angle of resolution; ISV, index of surface variance.

Regression analysis demonstrated significant treatment effects for UDVA and CDVA (Table 4 and Figure 7); 3.8 lines $(P<0.001)$ of improvement in UDVA and 1.5 lines $(P<0.001)$ of improvement in CDVA. The incremental trend over time for CDVA was equivalent to an improvement of about 1 line over a 3-year period $(P=0.067)$. The results in the keratoconus only group (Table 5) were similar and not statistically distinct from those in the whole group.

\section{Subjective Refraction}

At baseline, mean subjective astigmatism was $4.0 \pm 1.8$ diopters (D) (range 0.0 D to $8.0 \mathrm{D}$ ) and mean SEQ was $-4.2 \pm 4.6 \mathrm{D}$ (sphere range $-14.6 \mathrm{D}$ to $3.8 \mathrm{D}$ ). There was no indication of a consistent, significant change from baseline in subjective refraction parameters throughout followup (Tables 3 and 4).

\section{Keratometry}

No significant changes were reported in $\mathrm{K}_{\mathrm{m}}$, EKR $65 \mathrm{~K} 1$ and EKR $65 \mathrm{~K} 2$ in comparison with the preoperative baseline over the follow-up period. The $\mathrm{K}_{\mathrm{Max}}$ was $65.1 \pm 11 \mathrm{D}$. (range 46.3 D - 94.9 D) at baseline and showed a trend lower (flattening) throughout follow-up. The mean EKR global range was $24.2 \pm 10.4 \mathrm{D}$ (range $9.6 \mathrm{D}-49.0 \mathrm{D}$ ) at baseline and also trended lower throughout the follow-up period (Tables 2 and 3). 


\section{Improvement in Visual Acuity by Visit}

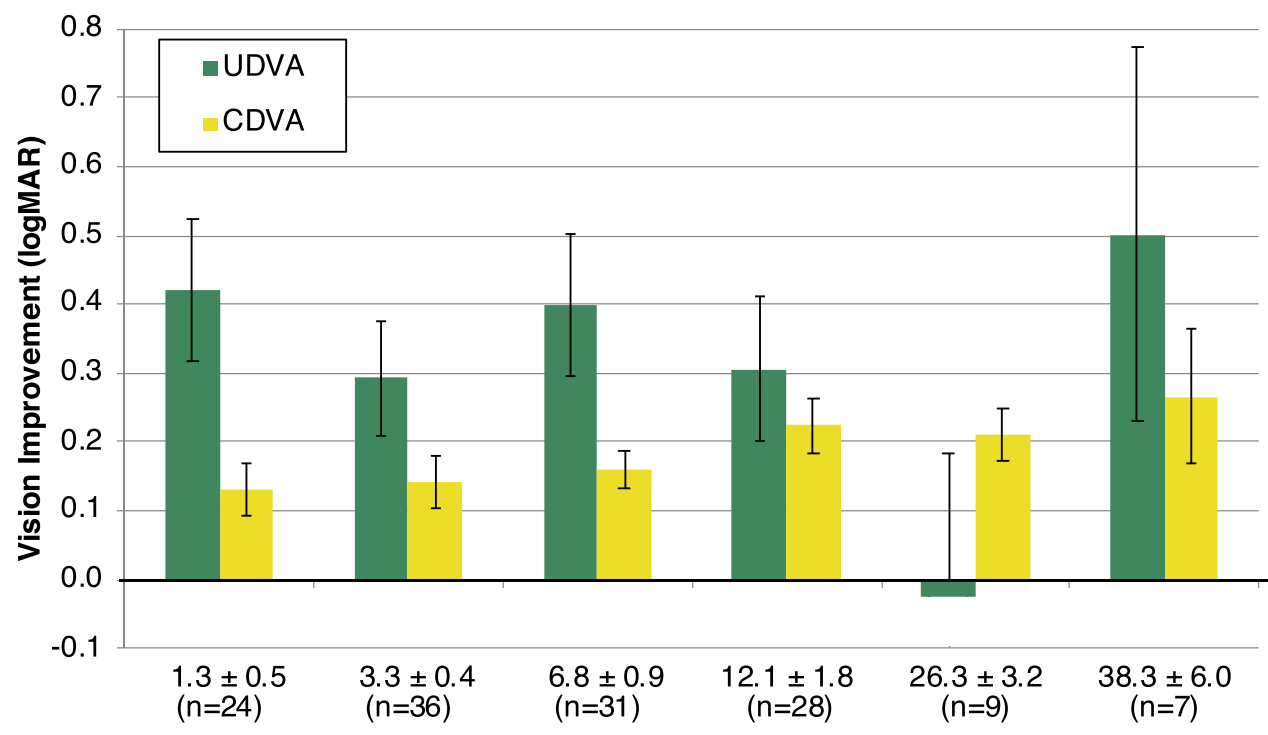

Time after $\mathrm{CK}+\mathrm{CXL}$ (months)

Figure 6 Chart showing mean ( \pm standard error) change in visual acuities by-visit.

\section{Tomographic Indices}

At baseline, the D index and ISV were $16 \pm 9$ (range 7-51) and $149 \pm 49$ (range 60-325), respectively (Table 2). At the postoperative visits, the mean ISV was lower than baseline, with the confidence intervals excluding zero at Visits 3-7 (Tables 2 and 3). Regression analysis demonstrated that, of the 9 tomographic parameters, only the D index and ISV showed significant trends (Table 4 and Figure 7). The D index shows a small and statistically equivocal $(P=0.1)$ treatment effect of $-1.4 \pm$ 0.8 , but a statistically significant $\left(P=3 \times 10^{-6}\right)$ progression of $2.2 \pm 0.5$ per year postoperative. The ISV shows a statistically significant $\left(P=7 \times 10^{-8}\right)$ treatment effect of $-28.5 \pm 5.0$, with a smaller and less significant $(P=0.01)$ rebound effect of $7.1 \pm$ 2.7 per year. The results in the keratoconus only group (Table 5 ) were similar and not statistically distinct from those of the entire cohort.

\section{Safety}

At the 1-year visit (Visit 6), there was no loss of $\geq 1$ line of CDVA in any eye. Three eyes (10.7\%) showed no change in lines of CDVA and all other eyes $(25 / 28,89.3 \%)$ demonstrated a gain of $1-8$ lines of CDVA. No eye lost $\geq 2$ lines of CDVA at their final visit, including those 3 to 4 years postoperative.

In 3 eyes, a transient microperforation of the cornea occurred and was repaired immediately with the application of ReSure Sealant (Ocular Therapeutix, Bedford, MA) with no sequelae. No haze, infection, or other adverse events occurred. Conductive keratoplasty spots fade over time and did not interfere with vision. No eyes exhibited any permanent apical scarring affecting vision.

\section{Discussion}

Conductive keratoplasty has been most commonly used for the treatment of hyperopia. In 2005, Alio et al were the first to describe $\mathrm{CK}$ for advanced $\mathrm{KC} .{ }^{28}$ Over the next 5 years, 3 other research groups ${ }^{19,25,29}$, published their results using CK to remodel the corneal shape of keratoconic eyes to reduce corneal irregularities and improve the quality of CDVA and visual function. Although these studies reported improvements in VA and contact lens tolerance after CK, the studies were limited by small numbers of patients (as few as 2) and follow-up as short as 1 month. The major issue with $\mathrm{CK}$ for $\mathrm{KC}$ was the rapid regression of effect, with both UDVA and CDVA decreasing toward preoperative values within 3 to 6 months. ${ }^{25}$

To mitigate the regression of effect after $\mathrm{CK},{ }^{25,30,31}$ we followed CK with a novel, epi-on, CXL technique that has demonstrated effectiveness in halting the progression of corneal ectasia. ${ }^{7}$ We found that timing of the CXL procedure after CK is critical. During our CK pilot trials for this study, it appeared that when CK and CXL were applied sequentially in the same session, considerable regression was observed. ${ }^{26} \mathrm{~A}$ similar effect has been reported by Cummings et al in combining another 
UDVA Change from Baseline

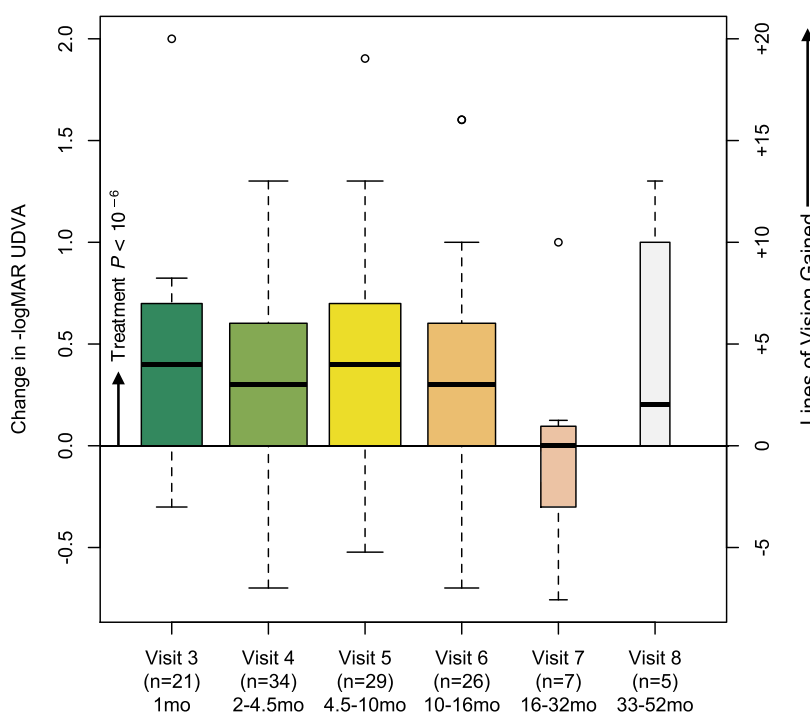

D Change from Baseline

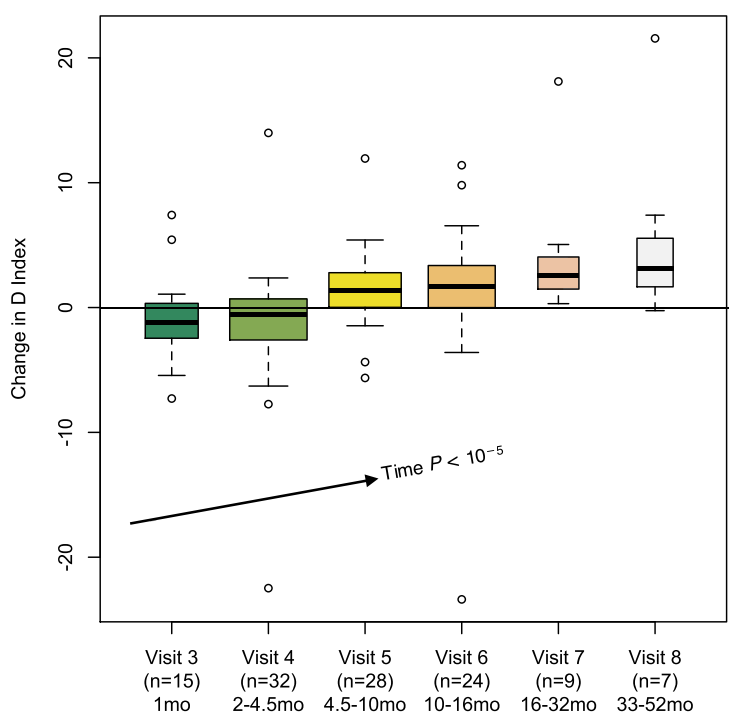

CDVA Change from Baseline

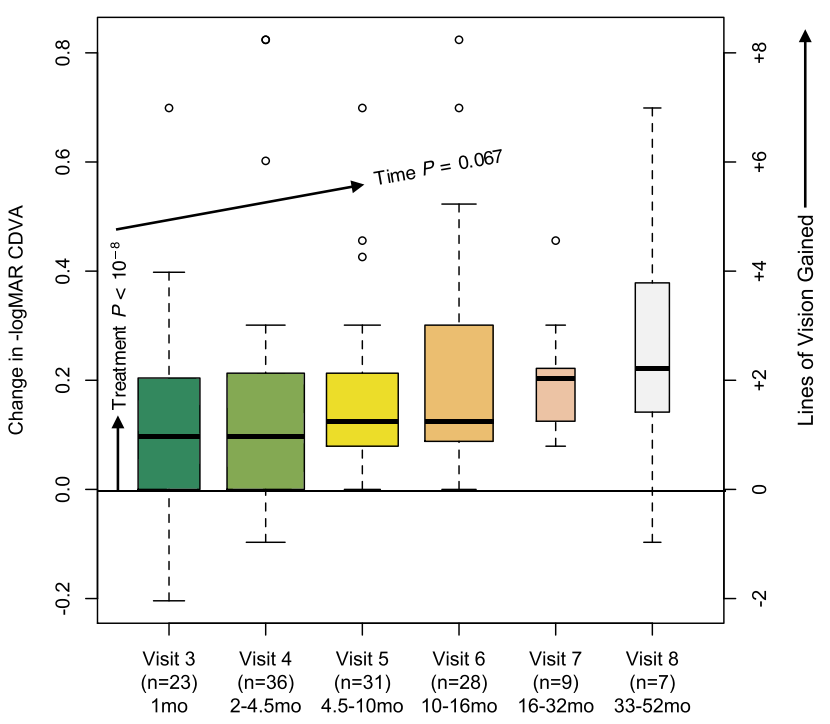

ISV Change from Baseline

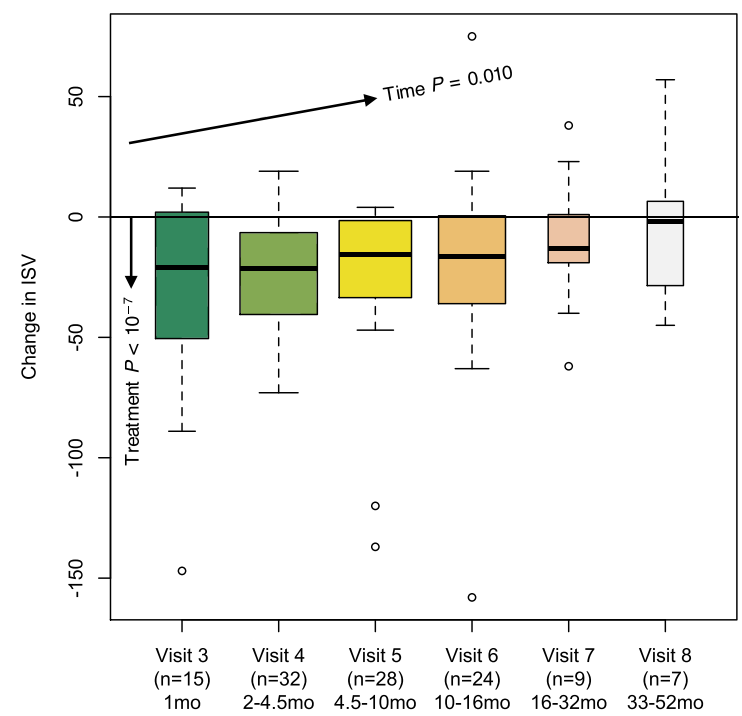

Figure 7 Distribution of change in visual acuity and tomographic indices by-visit. The boxplots summarize the observed data and illustrate the trends that demonstrated significant effects in the exploratory regression analysis. The dark horizontal line is median change. The top and bottom borders of the box indicate 75 th and 25 th percentiles, respectively. Whiskers indicate the full range. Dots indicate outliers ( $>1.5 x$ the interquartile range). Superimposed $P$ values describe the statistical strength of the effects returned by multiple regression analysis.

thermokeratoplasty technique (microwave) with CXL. ${ }^{32,33}$ Empirically, we had determined that a 1-day delay between CK and CXL provided the most consistent VA results over time.

This exploratory study suggests that the combination of apical spot placement CK followed 1 day later by epi-on CXL may improve and stabilize VA in patients with moderate to severe loss of CDVA due to keratoconus or post-LASIK ectasia. Both UDVA and CDVA showed substantial improvement and stability over the study period. The treatment effect on
CDVA, in particular, was highly significant overall (regression analysis) and continued to trend positive over time throughout the follow-up period of up to 3 years. Conversely, both SEQ and subjective astigmatism did not show any significant change. The poor correlation between objective measures, such as astigmatism magnitude and axis to values derived by subjective refraction has been previously documented. ${ }^{34}$ It has also been shown that objective measurements in ectatic eyes are inherently more variable ${ }^{35}$ and visual acuity results correlate poorly with curvature changes seen with intrastromal ring 
segments for keratoconus. ${ }^{36}$ Our study and others also showed a similar dissociation between visual acuity changes and keratometric changes after surgical interventions for keratoconus. $^{7}$

The combined procedure demonstrated a good safety profile. The only complication, micro-perforation of the cornea, which occurred early in the study, was remediated and prevented by a slight change to the angle of the CK probe during the procedure. All complications were rapidly resolved. There was no clinically meaningful regression in CDVA and no subsequent surgical management needed in any case. The safety was comparable to the demonstrated safety of transepithelial crosslinking. The same epi-on CXL technique used here was studied in 592 eyes without serious TEs or progression of disease. ${ }^{7}$

Historically, corneal topography has served to define, and follow the progression of, keratoconus. ${ }^{37,38}$ Thus, CXL studies have used a decrease in keratometric measures as a sign of treatment success in keratoconic eyes. ${ }^{6}$ In the current study, $\mathrm{K}_{\text {Max }}$ trended lower, most notably relative to baseline at Visits 4 and 5 , while $K_{m}$ varied and did not show any significant change over the study period. We also looked at EKR values, which more closely measure the optical power of the cornea than simulated keratometry; since they take into consideration both corneal surfaces and the existent back/front ratio in radii of curvature. The EKR are also more reliable in corneas that have undergone keratorefractive surgeries. ${ }^{39,40}$ However, the EKR parameters also did not show substantial change throughout follow-up except for a trend lower in the Global Range at Visits 6-8. These results are supported by the results of the linear regression analysis which showed no significant effect of treatment or time on these parameters.

In view of the VA improvements seen in the data it appears that common topographic and tomographic parameters useful in diagnosing and monitoring ectatic disease preoperatively may not be applicable post CK-CXL. After CXL for KC (without CK), poor correlations between tomographic indices and VAs have been documented. ${ }^{7,41,42}$ This may be attributed to higher-order distortions of the cornea in advanced ectasia. It has also been noted that CXL itself, in the short term, can scatter or modify the light paths of the Scheimpflug imaging system, leading to inaccurate images. ${ }^{43}$ The CK spots may do the same.

Apart from this, UDVA and CDVA remain paramount as outcome metrics, especially in advanced $\mathrm{KC}$, where the cornea is highly distorted. Reduced visual acuity is a major cause of morbidity and limitations in daily activities. Reshaping the cornea with $\mathrm{CK}$ can also lead to better contact lens tolerance, allowing a transition from specialized contact lenses to more standard lens designs, better spectacle tolerance and avoidance of corneal transplantation.

The current study demonstrated a 3-line improvement in UDVA and a 2-line improvement in CDVA at the 1-year visit in a population of patients with advanced vision loss due to ectasia. A pivotal clinal trial of standard CXL alone demonstrated an approximately 1-line improvement in UDVA and CDVA at 1-year postoperative. ${ }^{6}$ Using the same transepithelial protocol as the current study, Stulting reported a 1.5-line improvement in UDVA and a 1.5-line improvement in CDVA 1 and 2 years after CXL alone. In addition to VA improvements, higher order aberrations were reduced $28 \%$ at one year and $36 \%$ at two years. ${ }^{7}$

It has been suggested that the ISV, an anterior corneal index derived from Scheimpflug corneal imaging, may be a useful measure of corneal surface irregularity in the progression of keratoconus. Similar to VA, in this study the ISV index showed substantial improvement, particularly in the early follow-up visits, with minimal regression. Further study is necessary to determine whether the ISV is a reliable measure of vision-relevant irregularity of the cornea.

The D index shows some improvement but regresses over time. While D is a known predictor of keratoconus progression absent treatment, it is unknown how CK treatment would affect this metric. The separate effects of CXL and $\mathrm{CK}$ on this parameter would be of benefit in interpreting its utility for assessing disease status and prognosis post-treatment. The key outcome metric of vision is improved and supports treatment, but it may be possible for tomographic indices to indicate repeated crosslinking or other support to preserve VA long-term.

Keratoconus and other ectatic disorders are irregular corneal deformities, each case with its own clinical, pachymetric and tomographic characteristics. One advantage of this $\mathrm{CK}$ CXL technique is the ability of the surgeon to modify the original surgical plan based on the use of serial tomographic scans, including difference maps, peri-operatively to guide the placement of $\mathrm{CK}$ spots. Another is the minimally invasive nature of both CK and epi-on CXL, an advantage in these already highly compromised corneas.

A limitation of this report is its retrospective, exploratory nature. For this reason, the statistically significant results in vision and tomographic indices would benefit from confirmation in prospective multi-center randomized clinical trials. The number and timing of follow-up exams varied among patients. The regression analysis used time as a continuous variable in 
order to assess trends without bias due to binning measurements but does not address possible bias related to visit scheduling or loss to follow up. Multiple parameters were assessed in order to identify potentially predictive metrics, which increases the chance of Type 1 error (false positives). We did not make $P$-value corrections for multiple measures, but the treatment effects found only in CDVA, UDVA, and ISV, as well as the time-effect found on the $\mathrm{D}$ index would be considered statistically significant even with the most conservative $P$-value discounting, and should be studied further.

A further limitation, due to the retrospective/exploratory nature of the study, is that there is no control group. Corneal crosslinking has been associated with an approximately 1-line improvement in VA at 1-year post-procedure. ${ }^{6,7}$ Although the VA improvements in the current study were greater, further controlled trials are necessary to verify that $\mathrm{CK}$ with $\mathrm{CXL}$ improves VA results over CXL alone. We believe the current report supports the usefulness of continued study of this combination approach.

In summary, the current study describes a new technique that appears effective at improving and stabilizing vision over an intermediate period of follow-up (months to years) in eyes that had lost substantial CDVA due to corneal ectasia. Significant improvements in VA, the most important metric evaluated for alleviating morbidity and affecting patient satisfaction, ${ }^{44}$ were documented. Future study may be directed to the combination of non-invasive thermokeratoplasty techniques followed by this epi-on CXL treatment.

\section{Disclosure}

R.S. Rubinfeld equity interests in CXL Ophthalmics, LLC, Encinitas, CA, CurveRight, LLC, Bethesda, MD and CXLUSA, LLC, Bethesda, $\mathrm{M}$; in addition, Dr Roy S Rubinfeld has a patent US 9,622,911 licensed to CXLUSA, RSR, a patent US 9,555,111 licensed to CXLUSA, RSR, a patent EU 3,122,296 licensed to CXLUSA, RSR, a patent US 9,566,301 licensed to CXLUSA, RSR, a patent US 10,092,594 licensed to CXLUSA, RSR, a patent EU Appl'n 2,830,637 pending to CXLUSA, RSR, a patent US Appl'n 2019/0022134 pending to CXLUSA, RSR, a patent EU Appl'n 2,830,627 pending to CXLUSA, RSR, a patent US Appl'n 2014-0249509 pending to CXLUSA, RSR, a patent EU Appl'n 3,406,229 pending to CXLUSA, RSR, a patent US Appl'n 2017-0065826 pending to CXLUSA, RSR, a patent EU Appl'n 2,967,986 pending to CXLUSA, RSR. Dr. Parsons is a consultant for CXLO, LLC, Encinitas, CA. Dr. Belin has an equity interest in CXLO, LLC, Encinitas, $\mathrm{CA}$ and is a consultant for OCULUS GmbH, Wetzlar, Germany.
Dr Arthur B Cummings reports personal fees from Alcon, personal fees from WaveLight, personal fees from Vivior, personal fees from TearLab, personal fees from TearClear, personal fees from Scope Ophthalmics, personal fees from Allotex, outside the submitted work. The remaining authors have no financial interest to disclose.

\section{References}

1. Godefrooij DA, Mangen M-J-J, Chan E, et al. Cost-effectiveness analysis of corneal collagen crosslinking for progressive keratoconus. Ophthalmology. 2017;124(10):1485-1495. doi:10.1016/j. ophtha.2017.04.011

2. Cassidy D, Beltz J, Jhanji V, et al. Recent advances in corneal transplantation for keratoconus. Clin Exp Optom. 2013;96(2):165-172. doi:10.1111/cxo.12047

3. Chow S-P, Hopkinson CL, Tole DM, et al. Stability of visual outcome between 2 and 5 years following corneal transplantation in the UK. $\mathrm{Br}$ $J$ Ophthalmol. 2018;102(1):37-41. doi:10.1136/bjophthalmol-2016310129

4. Beckman KA, Gupta PK, Farid M, et al. Corneal crosslinking: current protocols and clinical approach. J Cataract Refract Surg. 2019;45 (11):1670-1679. doi:10.1016/j.jcrs.2019.06.027

5. Zhu AY, Jun AS, Soiberman US. Combined protocols for corneal collagen cross-linking with photorefractive surgery for refractive management of keratoconus: update on techniques and review of literature. Ophthalmol Ther. 2019;8(Suppl 1):15-31. doi:10.1007/ s40123-019-00210-3

6. Hersh PS, Stulting RD, Muller D, Durrie DS, Rajpal RK. United States multicenter clinical trial of corneal collagen crosslinking for keratoconus treatment. Ophthalmology. 2017;124(9):1259-1270. doi:10.1016/j.ophtha.2017.03.052

7. Stulting DR, Trattler WB, Woolfson JM, et al. Corneal crosslinking without epithelial removal. J Cataract Refract Surg. 2018;44 (11):1363-1370. doi:10.1016/j.jcrs.2018.07.029

8. Shaheen MS, Shalaby Bardan A, Pinero DP, et al. Wave front-guided photorefractive keratectomy using a high-resolution aberrometer after corneal collagen cross-linking in keratoconus. Cornea. 2016;35 (7):946-953. doi:10.1097/ICO.0000000000000888

9. Kanellopoulos AJ. Comparison of sequential vs same-day simultaneous collagen cross-linking and topography-guided PRK for treatment of keratoconus. J Refract Surg. 2009;25(9):S812-S818. doi:10.3928/1081597X-20090813-10

10. Tuwairqi WS, Sinjab MM. Safety and efficacy of simultaneous corneal collagen cross-linking with topography-guided PRK in managing low-grade keratoconus: 1-year follow-up. J Refract Surg. 2012;28 (5):341-345. doi:10.3928/1081597X-20120316-01

11. Kymionis GD, Grentzelos MA, Karavitaki AE, et al. Transepithelial phototherapeutic keratectomy using a 213-nm solid-state laser system followed by corneal collagen cross-linking with riboflavin and UVA irradiation. J Ophthalmol. 2010;2010:146543. doi:10.1155/2010/ 146543

12. Kapasi M, Baath J, Mintsioulis G, et al. Phototherapeutic keratectomy versus mechanical epithelial removal followed by corneal collagen crosslinking for keratoconus. Can J Ophthalmol. 2012;47 (4):344-347. doi:10.1016/j.jcjo.2012.03.046

13. Izquierdo LJ, Henriquez MA, McCarthy M. Artiflex phakic intraocular lens implantation after corneal collagen cross-linking in keratoconic eyes. J Refract Surg. 2011;27(7):482-487. doi:10.3928/ 1081597X-20101223-02

14. Yousif MO, Said AMA. Comparative study of 3 intracorneal implant types to manage central keratoconus. J Cataract Refract Surg. 2018;44(3):295-305. doi:10.1016/j.jcrs.2017.12.020 
15. Ertan A, Colin J. Intracorneal rings for keratoconus and keratectasia J Cataract Refract Surg. 2007;33(7):1303-1314. doi:10.1016/j.jcrs. 2007.02.048

16. Mounir A, Radwan G, Farouk MM, et al. Femtosecond-assisted intracorneal ring segment complications in keratoconus: from novelty to expertise. Clin Ophthalmol. 2018;12:957-964. doi:10.2147/OPTH.S166538

17. Brenner LF, Alio JL, Vega-Estrada A, et al. Indications for intrastromal corneal ring segments in ectasia after laser in situ keratomileusis. $J$ Cataract Refract Surg. 2012;38(12):2117-2124. doi:10.1016/j.jcrs 2012.07.036

18. Pinero DP, Alio JL. Intracorneal ring segments in ectatic corneal disease - a review. Clin Exp Ophthalmol. 2010;38(2):154-167. doi:10.1111/j.1442-9071.2010.02197.x

19. Kymionis GD, Kontadakis GA, Naoumidi TL, et al. Conductive keratoplasty followed by collagen cross-linking with riboflavin-UVA in patients with keratoconus. Cornea. 2010;29(2):239-243. doi:10.1097/ICO.0b013e3181a818ab

20. Charpentier DY, Bertel F, Duplessix M, et al. Hot needle thermal keratoplasty to correct naturally occurring hyperopic astigmatism. J Refract Surg. 1996;12(6):705-708.

21. Tutton MK, Cherry PM. Holmium:YAG laser thermokeratoplasty to correct hyperopia: two years follow-up. Ophthalmic Surg Lasers. 1996;27(5 Suppl):S521-S524.

22. Mannschreck DB, Rubinfeld RS, Soiberman US, et al. Diffuse lamellar keratitis after epi-off corneal crosslinking: an under-recognized complication? Am J Ophthalmol Case Rep. 2019;13:140-142. doi:10.1016/j.ajoc.2019.01.004

23. Rubinfeld RS, Epstein RH, Majmudar PA, et al. Transepithelial crosslinking retreatment of progressive corneal ectasia unresponsive to classic crosslinking. J Cataract Refract Surg. 2017;43(1):131-135. doi:10.1016/j.jcrs.2016.11.038

24. Coskunseven E, Kymionis GD, Tsiklis NS, et al. IG. Complications of intrastromal corneal ring segment implantation using a femtosecond laser for channel creation: a survey of 850 eyes with keratoconus. Acta Ophthalmol. 2011;89(1):54-57. doi:10.1111/ j.1755-3768.2009.01605.x

25. Kato N, Toda I, Kawakita T, et al. Topography-guided conductive keratoplasty: treatment for advanced keratoconus. Am J Ophthalmol. 2010;150(4):481-489.e1. doi:10.1016/j.ajo.2010.05.014

26. Cummings A, Sinjab M, Hatch K, et al. Combined corneal cross linking and other procedures: indications and application models. In: Sinjab M, Cummings A, editors. Corneal Collagen Cross Linking. Cham: Springer; 2017:87-165.

27. Rubinfeld RS, Stulting DR, Gum GG, et al. Quantitative analysis of corneal stromal riboflavin concentration without epithelial removal. J Cataract Refract Surg. 2018;44(2):237-242. doi:10.1016/j.jcrs. 2018.01.010

28. Alio JL, Claramonte PJ, Caliz A, et al. Corneal modeling of keratoconus by conductive keratoplasty. J Cataract Refract Surg. 2005;31 (1):190-197. doi:10.1016/j.jcrs.2004.10.042

29. Lyra JM, Trindade FC, Lyra D, et al. Outcomes of radiofrequency in advanced keratoconus. J Cataract Refract Surg. 2007;33(7): 1288-1295. doi:10.1016/j.jcrs.2007.03.042
30. Esquenazi S, He J, Kim DB, et al. Wound-healing response and refractive regression after conductive keratoplasty. J Cataract Refract Surg. 2006;32(3):480-486. doi:10.1016/j.jcrs.2005.12.077

31. Mohammadpour M, Heidari Z, Hashemi H. Updates on managements for keratoconus. J Curr Ophthalmol. 2018;30(2):110-124. doi:10. 1016/j.joco.2017.11.002

32. Cummings AB. Keraflex and corneal cross-linking for the treatment of keratoconus. Poster presented at: Irish College of Ophthalmologists Conference; May; 2011; Cavan, Ireland.

33. Cummings AB. Factors influencing the stability of keraflex treatments for keratoconus. Poster presented at: Annual Meeting of the American Academy of Ophthalmology; November; 2012; Chicago, Illinois.

34. Lee J, Belin M. Comparison of subjectively refracted cylinder, topographic cylinder, and topographic guided refractive cylinder in normal patients. Cornea. 1996;15(2):220. doi:10.1097/00003226-1996 03000-00035

35. Belin MW, Meyer JJ, Duncan JK, Gelman R, Borgstrom M, Ambrosio R. Assessing progression of keratoconus \& crosslinking efficacy: the Belin ABCD progression display. Int J Kerat Ect Cor Dis. 2017;6(1):1-10.

36. Carneiro AB, Carossa C, Paranhos A, Lottenberg CL. Correlation between the corneal curvature and improvement in visual acuity after intrastromal corneal rings implantation with intralase for keratoconus. Invest Ophthalmol Vis Sci. 2011;52:5165.

37. Choi JA, Kim M-S. Progression of keratoconus by longitudinal assessment with corneal topography. Invest Ophthalmol Vis Sci. 2012;53(2):927-935. doi:10.1167/iovs.11-8118

38. Sahin A, Yildirim N, Basmak H. Two-year interval changes in Orbscan II topography in eyes with keratoconus. J Cataract Refract Surg. 2008;34(8):1295-1299. doi:10.1016/j.jcrs.2008.04.016

39. Holladay JT. Accuracy of scheimpflug holladay equivalent keratometry readings after corneal refractive surgery. J Cataract Refract Surg. 2010;36(1):182-183; author reply 183-184. doi:10.1016/j.jcrs.20 09.09 .010

40. Holladay JT. Holladay Report Interpretation Guidelines 2016. Oculus; 2016:1-14.

41. Kasai K, Kato N, Konomi K, et al. Flattening effect of corneal cross-linking depends on the preoperative severity of keratoconus. Medicine. 2017;96(40):e8160. doi:10.1097/MD.0000000000008160

42. Greenstein SA, Fry KL, Hersh PS. Corneal topography indices after corneal collagen crosslinking for keratoconus and corneal ectasia: one-year results. J Cataract Refract Surg. 2011;37(7):1282-1290. doi:10.1016/j.jcrs.2011.01.029

43. Belin MW, Khachikian S. Understanding elevation based topography: how elevation data is displayed. In: Belin MW, Khachikian S, Ambrosio R Jr, editors. Elevation Based Corneal Tomography. 2nd ed. City of Knowledge: Jaypee-Highlights Medical Publisher, Inc; 2012:25-45.

44. Tan JCK, Nguyen V, Fenwick E, et al. Vision-related quality of life in keratoconus: a save sight keratoconus registry Study. Cornea. 2019;38(5):600-604. doi:10.1097/ICO.0000000000001899
Clinical Ophthalmology

\section{Publish your work in this journal}

Clinical Ophthalmology is an international, peer-reviewed journal covering all subspecialties within ophthalmology. Key topics include: Optometry; Visual science; Pharmacology and drug therapy in eye diseases; Basic Sciences; Primary and Secondary eye care; Patient Safety and Quality of Care Improvements. This journal is indexed on PubMed
Central and CAS, and is the official journal of The Society of Clinical Ophthalmology (SCO). The manuscript management system is completely online and includes a very quick and fair peer-review system, which is all easy to use. Visit http://www.dovepress.com/ testimonials.php to read real quotes from published authors. 